\title{
Caracterização da Colônia de Pescadores Z2 de Cáceres em Mato Grosso
}

\author{
Characterization of the Z2 Angler Colony in Cáceres in Mato Grosso
}

\section{Caractérisation de la Colonie de Pêcheurs Z2 de Cáceres dansl'étatdu Mato Grosso}

\author{
Caracterización de Colonia Z2 de Pescadores de Cáceres en Mato Grosso \\ Cristiane Lima Façanha* \\ Carolina Joana da Silva*
}

Recebido em 28/04/2016; revisado e aprovado em 03/08/2016; aceito em 06/10/2016

DOI: http:/ / dx.doi.org/10.20435/1984-042X-2017-v.18-n.1(10)

\begin{abstract}
Resumo: Esta pesquisa foi realizada na Colônia de pescadores Z2, em Mato Grosso, com o objetivo de caracterizá-la por meio de pesquisa documental. A Colônia é composta por 692 pescadores, com idade entre 30 e 60 anos e tempo de pesca de menos de 10 anos. A análise evidenciou a crescente participação das mulheres atuando como pescadoras e gestoras administrativas da Colônia. A base de dados apresentou-se como um recurso para subsidiar os pescadores nas demandas de sua inclusão em políticas públicas.
\end{abstract}

Palavras-chave: Pantanal; Amazônia; pesca.

Abstract: This survey was conducted in order to characterize the Z2 angler Colony in Mato Grosso, through documentary research. The Z2 Colony consists of 692 members, aged between 30 and 60 years old, fishing within less than 10 years. The analysis revealed the increasing participation of women as anglers and managers of the Colony. The database was presented as a resource to subsidize anglers on the demands of their inclusion in public policies.

Key words: Pantanal; Amazônia; fishing.

Résumé: La presente recherche a été réalisée au sein de la Colonie de Pêcheurs Z2, dans le Mato Grosso, avec pour objectif la caractérisation de la Colonie au moyen d'une recherche documentaire. La Colonie comporte 692 pêcheurs, de 30 a 60 ans et dont l'activité de pêche date de moins de 10 ans. L'analyse a mis en évidence la participation croissante des femmes comme pêcheuses et gestionnaires administratives de la Colonie. La base de données s'est révelée être une ressource utile pour appuyer les pêcheurs dans leurs demandes d'insertion dans les politiques publiques.

Mots-clés: Pantanal; Amazônia; pêche.

Resumen: Esta encuesta se realizó junto a los pescadores de la Colonia Z2, en Mato Grosso, con el fin de caracterizar a través de la investigación documental. La colonia se compone de 692 pescadores, con edades comprendidas entre 30 y 60 años y el tiempo de pesca de menos de 10 años. El análisis mostró que el aumento de la participación de las mujeres que actúan como pescadores y gestores administrativos de Colonia. La base de datos se presenta como un recurso para apoyar a los pescadores en las demandas de su inclusión en las políticas públicas.

Palabras clave: Pantanal; Amazônia; pesca.

\section{INTRODUÇÃO}

De acordo com a Constituição Brasileira de 1988, a pesca profissional no Brasil é organizada por representações que vão desde a Confederação Nacional de Pescadores, Federação Estadual e Colônias de Pescadores. Essas Colônias foram equiparadas aos sindicatos urbanos, incluindo aí os princípios de livre organização, a não interferência do poder público sobre a organização sindical, o fim da filiação compulsória, a autonomia dos sindicatos e a unicidade sindical dentro de um município (BRASIL, 1988). As Colônias são organizações sociais que

* Universidade do Estado de Mato Grosso, Cáceres, Mato Grosso, Brasil.

INTERAÇÕES, Campo Grande, MS, v. 18, n. 1, p. 129-136, jan./mar. 2017. 
representam a classe dos pescadores no sentido de intervir a favor da atividade junto ao governo brasileiro, desde 1846 (BEGOSSI, 2002).

No Estado de Mato Grosso, existem 21 colônias de pescadores profissionais, distribuídas nas três bacias hidrográficas, Amazônica, Araguaia-Tocantins e Paraguai, onde atuam cerca de 14 mil pescadores. Essas colônias são regidas pela Lei de Pesca (Lei $n^{0}$ 6.672), que dispõe sobre a Política da Pesca no Estado de Mato Grosso, promulgada em 1995, a qual definiu que tanto a pesca amadora como a pesca profissional só podem ser exercidas de forma artesanal, que se expressa através da proibição de praticamente todos os petrechos de pesca, ficando, nesse momento, permitida somente a pesca com anzol e vara ou linha de mão.

Em 2009, a lei da pesca (Lei n ${ }^{\circ} 9.096$ ) foi alterada apresentando duas considerações sobre pesca profissional: a primeira afirma que a "pesca profissional artesanal é aquela exercida por pescadores profissionais que, com meios de produção próprios, exercem sua atividade de forma autônoma, individualmente ou em regime de economia familiar, ou ainda com o auxílio eventual de outros parceiros, sem vínculo empregatício" e a segunda, define a pesca profissional como "aquela praticada por pescadores que fazem da pesca sua profissão ou meio principal de vida" (MATO GROSSO, 2009).

Estudos realizados com colônia de pescadores em Mato Grosso abordam aspectos sociais e percepção ambiental (MEDEIROS, 1999; BEZERRA e OLIVEIRA, 2011; TOCANTINS et al., 2011; ARRUDA et al., 2014; SILVA et al., 2014). No entanto ainda existem lacunas na caracterização socioambiental mais abrangente das colônias. Dessa forma, esta pesquisa visa caracterizar a Colônia de Pescadores Z2 de Cáceres, MT, para dar suporte a uma participação qualificada na tomada de decisões junto às esferas governamentais.

\section{2 ÁREA DE ESTUDO}

A Colônia de Pescadores Z2, com sede em Cáceres, tem como área de atuação de seus filiados duas bacias hidrográficas: a do Alto Rio Paraguai, onde a atividade de pesca é desenvolvida no Rio Paraguai e seus afluentes a montante dessa cidade, e a bacia do Alto Rio Guaporé, onde a pesca é desenvolvida principalmente no rio Guaporé, com uma coordenação da colônia em Vila Bela da Santíssima Trindade (Figura 1). 


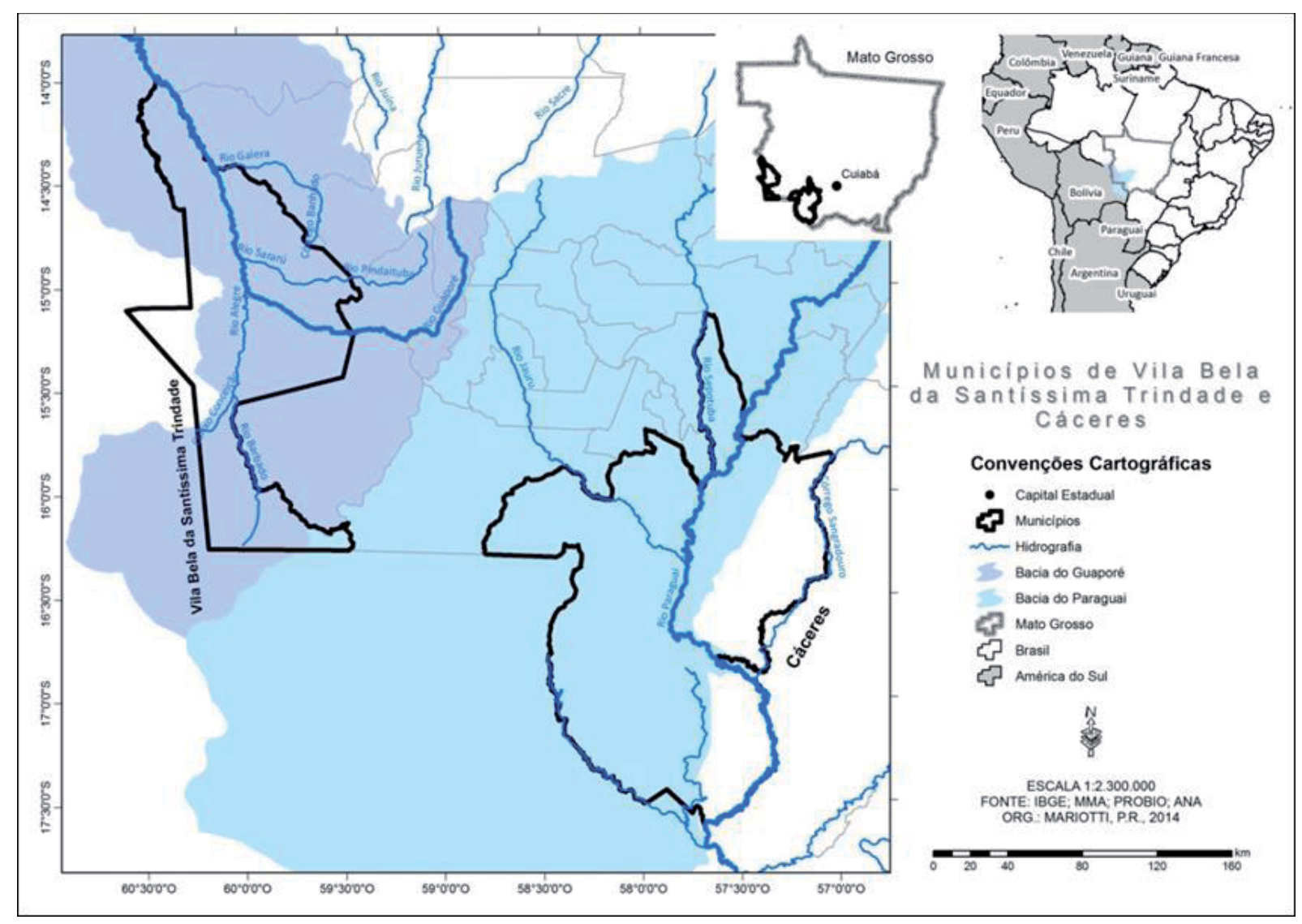

Figura 1 - Localização dos municípios de Cáceres e Vila Bela da Santíssima Trindade, Mato Grosso.

Fonte: Elaborado por Paulo Roberto Mariotti

\section{METODOLOGIA}

A caracterização da Colônia Z2 foi realizada por meio de pesquisa do tipo documental, baseada em todas as fichas dos sócios da Colônia, com cujo presidente obteve-se a devida autorização. Pesquisa documental "é aquela realizada a partir de documentos, contemporâneos ou retrospectivos, considerados cientificamente autênticos" (PÁDUA, 1997, p. 62). A riqueza de informações encontradas em documentos possibilita o entendimento de objetos que necessitam de contextualização histórica e sociocultural (CELLARD, 2008).

\section{RESULTADOS E DISCUSSÃO}

As Colônias de pescadores em Mato Grosso foram fundadas no início da dé- cada de 1980 pela Superintendência de Desenvolvimento da Pesca (SUDEPE) e, após sua extinção, passaram a ser de responsabilidade do IBAMA (Instituto Brasileiro do Meio Ambiente e dos Recursos Naturais Renováveis), que assumiu toda a gestão da pesca no território brasileiro (MEDEIROS, 1999).

Pela legislação pesqueira vigente no Estado de Mato Grosso (Lei n ${ }^{\circ}$ 9.096, de 16 de janeiro de 2009), somente é permitida a pesca artesanal, a qual é desenvolvida em sua quase totalidade de forma individual, em pequenas canoas ou no máximo com um barco motorizado de pequena potência. Tem-se aqui uma situação diferente do que ocorre com a pesca em águas interiores na Amazônia, onde, via de regra, a pesca profissional se caracteriza pela atividade em grandes barcos, com os pescadores trabalhando para os proprietários 
destes (MEDEIROS, 1999).

Criada em 03 de junho de 1982, através da portaria n. 046 da Confederação Nacional dos Pescadores, a Colônia de Pescadores Z2, atualmente conta com 692 filiados, residentes em nove municípios do Estado de Mato Grosso: Cáceres, Mirassol D'Oeste, Curvelândia, Porto
Esperidião, São José dos Quatro Marcos, Nova Lacerda e Glória d'Oeste, pertencentes à bacia hidrográfica do Alto Rio Paraguai e Vila Bela da Santíssima Trindade e Pontes e Lacerda, na bacia Amazônica. Destes 692 filiados, 473 são pescadores do sexo masculino e 219 do sexo feminino (Figura 2).

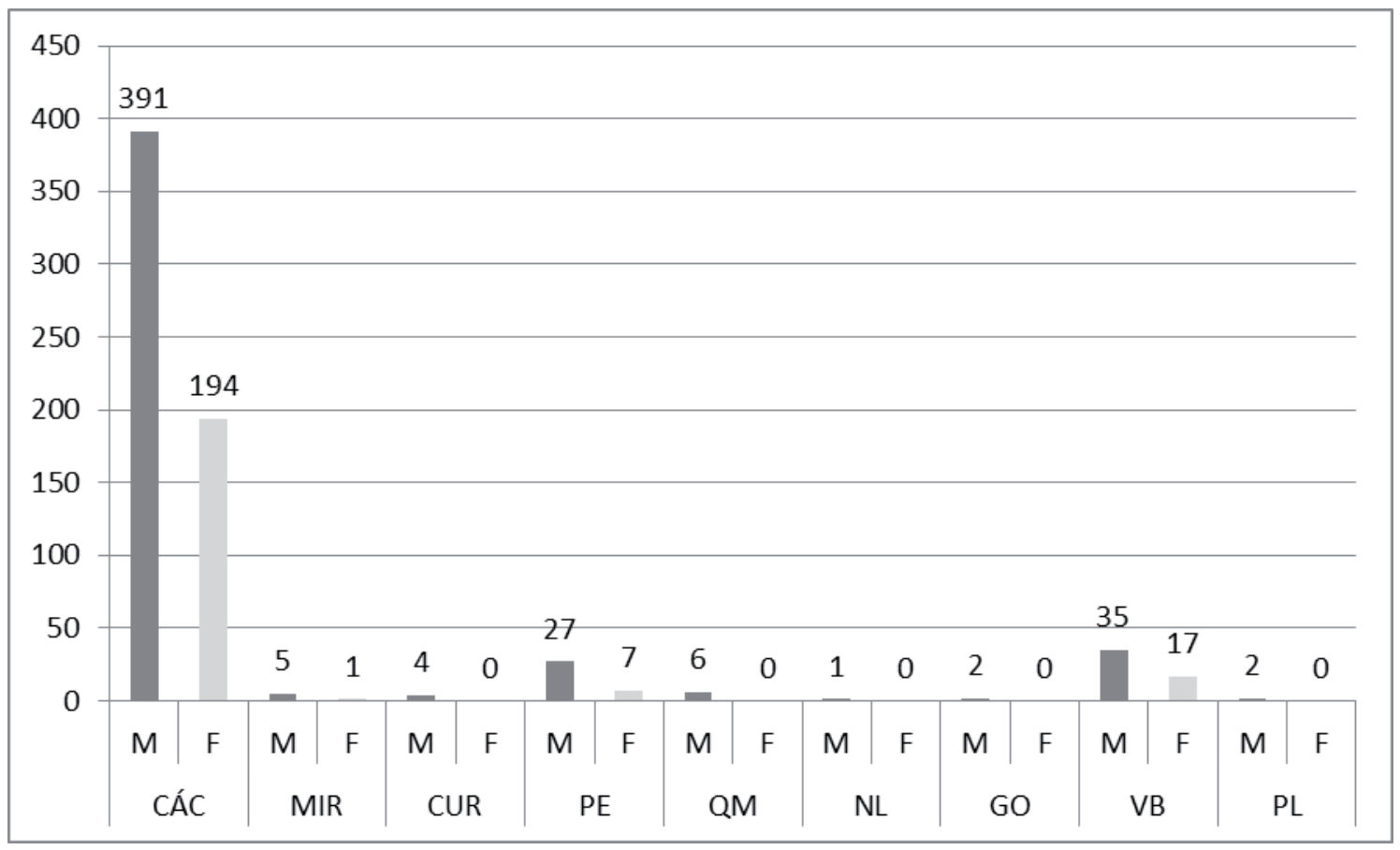

Figura 2 - Composição da Colônia Z2 com relação ao gênero

Legenda: CÁC: Cáceres; MIR: Mirassol d'Oeste; CUR: Curvelândia; PE: Porto Esperidião; QM: São José dos Quatro Marcos; NL: Nova Lacerda; GO: Glória d'Oeste; VB: Vila Bela da Santíssima Trindade; PL: Pontes e Lacerda. M: masculino; F: feminino.

No Pantanal diversos estudos mostraram a predominância do gênero masculino em atividades pesqueiras (COSTA JR., 1993; DA SILVA; SILVA, 1995; PORTO, 1999; MEDEIROS et al., 2000; SILVA et al., 2014), sendo que o mesmo ocorre na Amazônia nos trabalhos descritos por Ribeiro e Petrere Jr. (1990), Leme da Silva e Begossi (2004), Doria et al. (2008).

Ainda que as pesquisas mostrem maior participação de homens na pesca, a presença de mulheres na Colônia Z2 já foi observada desde 2010, quando foram registrados 543 pescadores profissionais, dos quais 422 pertenciam ao sexo mas- culino e 121 ao feminino (SILVA et al., 2014). Os resultados da presente pesquisa evidenciam um aumento no número total de pescadores, de 543 para 692, ou seja, 149 novos pescadores filiados, sendo 51 homens e 98 mulheres.

Além do aumento da participação das mulheres na pesca, elas também assumem a organização e a liderança da Colônia. Atualmente, a presidência da Colônia Z2 e a coordenação em Vila Bela da Santíssima Trindade são lideradas por mulheres, evidenciando a sua participação na pesca profissional e nos espaços institucionais da Colônia. 
Esse resultado corrobora com Vasconcellos et al. (2007) por registrarem um maior papel das mulheres na organização dos profissionais de pesca. Corrobora também com Leitão (2010) ao mostrar que, nas últimas décadas, as diretorias de pesca têm sido cada vez mais ocupadas por mulheres, devido às mudanças sociais que resultam na valorização e conhecimento de seus direitos.

Acredita-se que a atuação das mulheres como pescadoras possa estar relacionada aos benefícios sociais associados a essa atividades, como é o caso do seguro desemprego, pago aos pescadores profissionais durante o período do Defeso, que ocorre entre novembro e fevereiro, no intuito de contribuir com o aumento da renda familiar dos pescadores profissionais, bem como com a possibilidade de realizar a tarefa junto com o marido, aumentando o esforço de pesca e consequentemente da produtividade.

Com relação à idade dos pescadores, verificou-se uma variação entre 20 e 89 anos, sendo importante observar que a maioria dos pescadores tem idade entre 30 e 59 anos de idade (Figura 3).

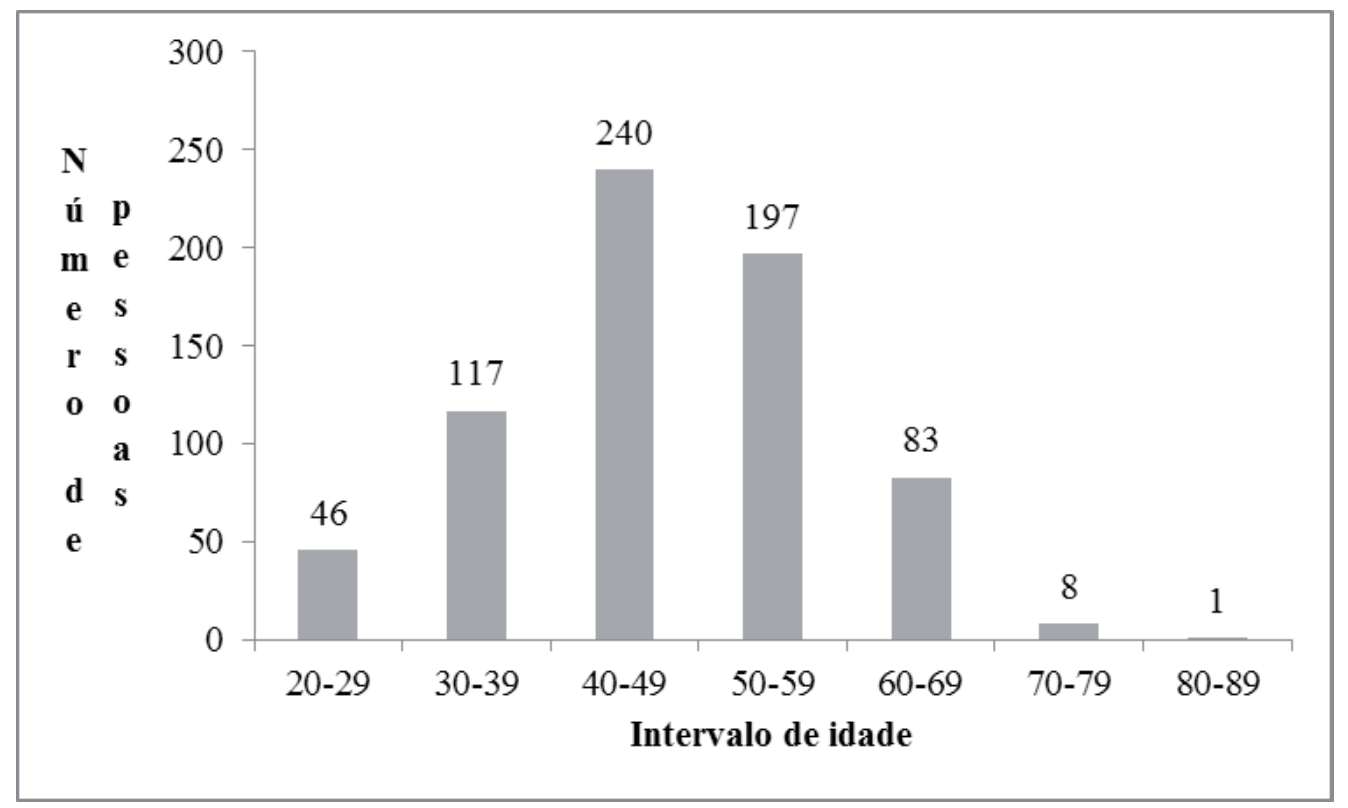

Figura 3 - Faixa etária dos pescadores da Colônia Z2

Os resultados desta pesquisa corroboram com a situação etária dos pescadores no Brasil, pois, de acordo com o Ministério da Pesca e Aquicultura (BRASIL, 2011), 77,8\% dos pescadores profissionais do país possuem 30 anos ou mais, enquanto que $22,2 \%$ dos pescadores têm menos de 30 anos de idade.
Com relação ao tempo de atuação profissional nas atividades de pesca, a maioria dos pescadores filiados à Colônia Z2 está há pouco tempo, entre 1 a 10 anos (Figura 4). Ressalta-se aqui que 60 fichas dos associados à Colônia Z2 não continham essa informação. 


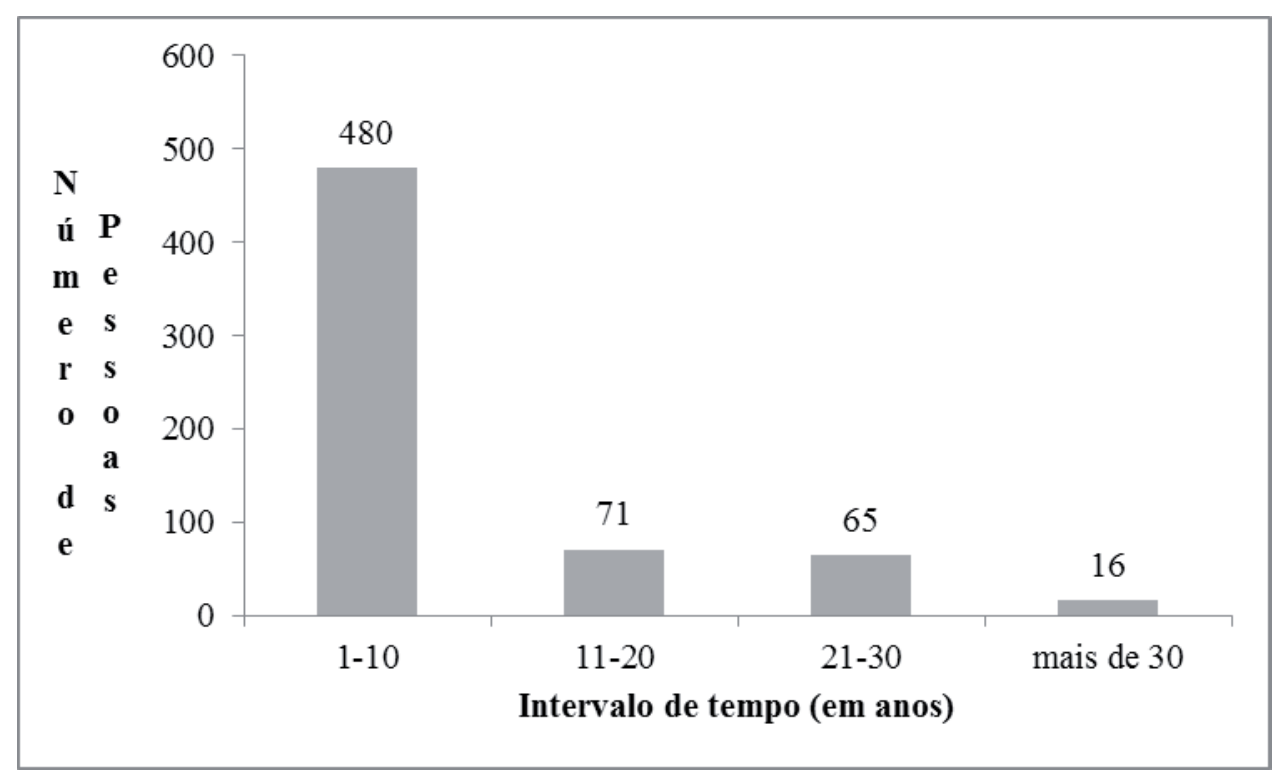

Figura 4 - Tempo de atuação dos pescadores como profissionais

O tempo de atuação na pesca dos pescadores da Colônia Z2, que vivem em área urbana, é diferente dos pescadores filiados a Colônia Z5, no município de Barão de Melgaço, que vivem, na maioria das vezes, na zona rural e organizados em comunidades tradicionais, os quais apresentam um período maior de vivência na pesca (MORAIS et al., 2012). O fato de mais pessoas terem pouco tempo de registro na atividade pesqueira, na Colônia Z2, pode estar relacionado ao pagamento de seguro-defeso pelo governo federal, como forma de incentivo à filiação do pescador à colônia no intuito de obter uma melhoria na renda familiar. De acordo com Lourenço et al. (2004), o seguro pode ser perdido em função de algumas condições como recebimento de aposentadoria, de salário oriundo de outra atividade que não a pesqueira ou, ainda, em caso de o pescador utilizar empregados em sua atividade de pesca.

Ainda que a atuação como pescador profissional seja recente, muitos pescadores em Cáceres praticam a atividade pesqueira bem antes da exigência legal da carteira profissional de pesca, e alguns destes pescam no rio Paraguai desde sua infância. A atividade pesqueira é praticada em toda a extensão urbana do rio, na cidade de Cáceres, onde, nos meses mais propícios à pesca (abril-setembro), as margens do rio Paraguai apresentam-se como um espaço coletivo e solidário. É quando todas as funções ecológicas do Pantanal emergem, mediadas pelos serviços ecossistêmicos de regulação, por meio do pulso de inundação; de provisão, pela garantia de suas condições ecológicas, para a reprodução dos peixes; de produção, sustentando a renda familiar de pescadores; e de cultural/informação, na oferta de lazer, turismo e educação, propícios à realização desta pesquisa.

\section{CONSIDERAÇÕES FINAIS}

Colônias de pescadores foram criadas no intuito de representar favoravelmente os pescadores junto ao governo brasileiro. A Colônia Z2 em Mato Grosso pode ser considerada um espaço de discussão de estratégias de manejo e conservação dos recursos pesqueiros, para conectar ações similares no Pantanal e na Amazônia, visto que seus filiados atuam principalmente nos rios Paraguai e Guaporé.

A base de dados organizada e mantida pela Colônia Z2 permitiu a sua análise documental, a qual mostrou o papel e o estado atual da pesca, na garantia 
do sustento de pescadores profissionais, homens e mulheres, jovens, adultos e idosos, revelando ainda a atuação social de mulheres no desempenho de novas responsabilidades na obtenção de renda e na gestão da colônia. Além disso, ao servir de referência para este e outros futuros estudos, a base de dados apresentou-se como um recurso que pode subsidiar os pescadores nas suas demandas de inclusão socioambiental nas políticas públicas que atendam seus interesses.

\section{REFERÊNCIAS}

ARRUDA, Selma Beatriz Sala de; ANDRADE, Leila Nalis Paiva da Silva; SOUZA, Célia Alves de; CRUZ, Jean da Silva; LEANDRO, Gustavo Roberto dos Santos. Características socioeconômicas dos ribeirinhos no rio Paraguai, município de Cáceres, Pantanal Mato-Grossense - Brasil. Geografia em Questão, Marechal Cândido Rondon, v. 07, n. 2, p. 162177, 2014.

BEGOSSI, Alpina. Latin América Fisheries: local organizationand management. Tunisia: Latin América Fisheries, ISEE, 2002. p. 6-9.

BEZERRA, Darci Ordonio dos Santos; OLIVEIRA, Haydee Torres de. Impactos socioambientais no rio Paraguai, Cáceres, Mato Grosso, Brasil: percepção dos pescadores da Colônia Z-2. Ciência e Educação, Bauru, v. 17, n. 4, p. 957-973, 2011.

BRASIL. Ministério da Pesca e Aquicultura. Boletim Estatístico da Pesca e Aquicultura. Brasília, DF: MPA, 2011. Disponível em < http:/ / www. mpa.gov.br/files/docs/Boletim_MPA_2011_ pub.pdf>. Acesso em: 4 jun. 2015.

Constituição da República Federativa do Brasil. Brasília, DF: Senado Federal, 1988.

CELLARD, André. A análise documental. In: POUPART, J. et al. A pesquisa qualitativa: enfoques epistemológicos e metodológicos. Petrópolis: Vozes, 2008.

COSTA JR., Plácido. Os vilões do Cuiabá: um estudo sobre a pesca em Estirão Comprido - Pantanal de Barão de Melgaço - MT. 1993. Monografia (Especialização em Educação Ambiental) - Universidade Federal de Mato Grosso, Cuiabá, MT, 1993.
DA SILVA, Carolina Joana; SILVA, Joana Aparecida Fernandes. No ritmo das águas do Pantanal. São Paulo: NUPAUB/USP, 1995. 210 p.

DÓRIA, Carolina Rodrigues da Costa; ARAÚJO, Túlio Raimundo de; SOUZA, Suelen Taciane Brasil de; TORRENTE-VILARA, Gislene. Contribuição da etnoictiologia à análise da legislação pesqueira referente ao defeso de espécies de peixes de interesse comercial no oeste da Amazônia Brasileira, rio Guaporé, Rondônia, Brazil. Biotemas, Florianópolis, v. 21, n. 2, p. 119-132, 2008.

LEITÃO, Maria do Rosário de Fátima Andrade. Gênero e pesca: 30 anos de registro geral da pesca. In: FAZENDO GÊNERO - DIÁSPORA, DIVERSIDADES, DESLOCAMENTOS, 9., 23-26 ago. 2010, Florianópolis. Anais... Florianópolis: UFSC, 2010.

LEME DA SILVA, Andréa; BEGOSSI, Alpina. Uso dos recursos por ribeirinhos no Médio Rio Negro. In: BEGOSSI, A. Ecologia de pescadores da Mata Atlântica e da Amazônia. São Paulo: Nupaub/USP; FAPESP, 2004. 332 p.

LOURENÇO, Celeste Ferreira; HENKEL, Jimnah de Almeida e Silva; MANESCHY, Maria Cristina Alves. A seguridade social para os pescadores artesanais no Brasil: estudo de Caso no Pará. Monografia Samudra. Belém: Coletivo Internacional de Apoio aos Trabalhadores da Pesca (ICSF), 2004.

MATOGROSSO. Lei $n^{\circ}$ 9.096, de 16 de janeiro de 2009. Dispõe sobre a Política da Pesca no Estado de Mato Grosso e dá outras providências. Cuiabá: Assembleia Legislativa, 2009.

MEDEIROS, Heitor Queiroz de; AMARAL, Viviane Lucas do; MATEUS, Lucia Aparecida de Fátima; PENHA, Jerry Magno Ferreira; DA SILVA, Carolina Joana. Caracterização da Atividade de Pesqueira Comercial no Alto Rio Paraguai, Município de Barra do Bugres, Mato Grosso. In: SIMPÓSIO SOBRE RECURSOS NATURAIS E SÓCIO-ECONÔMICOS DO PANTANAL: OS DESAFIOS DO NOVO MILÊNIO, 3., 27-30 nov. 2000. Corumbá, MS. Anais... Corumbá: EMBRAPA, 2000.

MEDEIROS, Heitor Queiroz de. Impactos das politicas públicas sobre os pescadores profissionais do Pantanal de Cáceres - Mato Grosso. 1999. Dissertação (Mestrado em Cência Ambiental) - Universidade de São Paulo, PROCAM, São Paulo, SP, 1999. 
MORAIS, Fernando Ferreira de; IGNES, Juliano Ribas; DA SILVA, Carolina Joana. Uma abordagem etnobiológica da comunidade Estirão Comprido, entorno do sistema de Baías Chacororé-Sinhá Mariana. In: DA SILVA, Carolina Joana; SIMONI, Jane (Org.). Água, biodiversidade e cultura do Pantanal: estudos ecológicos e etnobiológicos no sistema de Baías Chacororé-Sinhá Mariana. Cáceres: Ed. UNEMAT, 2012. p. 155-168.

PÁDUA, Elisabete Matallo Marchesini de. O processo de pesquisa. Metodologia da pesquisa: abordagem teórico-prática. Campinas: Papirus. 1997. p. 29-89.

PORTO, Ricardo de Andrade. Diagnóstico sócio-econômico-ambiental da pesca no Pantanal de Barão de Melgaço, Mato Grosso: um estudo de caso. 1999. Dissertação (Mestrado em Ecologia) - Universidade Federal de Mato Grosso, Cuiabá, MT, 1999.

RIBEIRO, Mauro Cesar Lambert de Brito; PETRERE JR., Miguel. Fisheries and management of the jaraqui (Semaprochilodustaeniurus, S. insignis). Central Amazônia Reseach e Management, v. 5, p. 195-215, 1990.

SILVA, Rosimeire Vilarinho da; SOUZA, Célia Alves de; BAMPI, Aumeri Carlos. Os olhares dos pescadores profissionais e proprietários comerciais, sobre o Rio Paraguai em Cáceres, Mato Grosso. Revista Brasileira de Ciências Ambientais, Rio de Janeiro, n. 32, p. 24-41, jun. 2014.

TOCANTIS, Nely; ROSSETTO, Onélia Carmem; BORGES, Flavyane Rosa. Abordagem socioeconômica dos pescadores filiados à colônia Z11: município de Poconé, Pantanal de Mato Grosso, Brasil. Revista Geográfica de América Central, Heredia, p. 01-09, 2011.

VASCONCELLOS, Marcelo; DIEGUES; Antônio Carlos S. A; SALES, Renato Rivaben. Limites e possibilidades na gestão da pesca artesanal costeira. In: COSTA, Adriane Lobo (Org.). Nas redes da pesca artesanal. Brasília: IBAMA/MMA, 2007. p. 15-83.

\section{Sobre as autoras:}

Cristiane Lima Façanha: Doutora em Biodiversidade e Biotecnologia pela Rede de Biodiversidade e Biotecnologia da Amazônia Legal (Rede BIONORTE), mestrado em Ciências Ambientais pela Universidade do Estado de Mato Grosso, graduação em Ciências Biológicas pela Universidade Federal de Mato Grosso. E-mail: crisfacanha@gmail.com

Carolina Joana da Silva: Pós Doc em Limnologia de Áreas Úmidas Tropicais, no Grupo de Ecologia Tropical do Instituto Max Planck de Limnologia, Plom, Alemanha, doutorado em Ecologia e Recursos Naturais pela Universidade Federal de São Carlos (UFSCAR), mestrado em Biologia (Ecologia) pelo Instituto Nacional de Pesquisas da Amazônia (INPA) e graduação em Licenciatura e História Natural pela Universidade Federal de Mato Grosso (UFMT). Professora Adjunta da Universidade do Estado de Mato Grosso (UNEMAT). E-mail: ecopanta@terra.com.br 